$\underline{\text { Review }}$

\title{
An overview of human infections caused by Staphylococcus pseudintermedius: A zoonotic risk of the oldest friend
}

\author{
MAR Priyantha \\ Sri Lankan Journal of Infectious Diseases 2022 Vol.12(1):E1 1-8 \\ DOI: http://dx.doi.org/10.4038/sljid.v12i1.8415
}

\begin{abstract}
Staphylococcus pseudintermedius is colonized in $90 \%$ of healthy dogs and the infection has also been reported in humans who have close contact with dogs. S. pseudintermedius is a Gram positive coagulase positive coccus which is an opportunistic pathogen. Human infection has been reported less frequently either due to misidentification or misdiagnosis or to underreporting. The objective of this overview is to highlight the epidemiology, clinical presentation, importance of laboratory tests and antimicrobial resistance of $S$. pseudintermedius in humans.
\end{abstract}

Human infections of $S$. pseudintermedius have been reported as case studies or clusters of infections in the literature. Several cases have been reported among the adult population, and in patients with metabolic diseases such as diabetes mellitus, immunocompromised states such as AIDS, cancers, and autoimmune diseases. The organism had been isolated and identified both in healthy people and individuals with adverse clinical outcomes. S. pseudintermedius causes skin and soft tissue infections, catheter associated infections and surgical site infections in humans. The incidence of skin and soft tissue infection by S. pseudintermedius in humans was $0.025 \%$ in Canada. Methicillin resistance $S$. pseudintermedius (MRSP) has been isolated both in healthy people and associated with clinical infections. MRSP is often multidrug resistant and therapeutic options are limited. Multidrug resistance has been detected in methicillin susceptible $S$. pseudintermedius (MSSP). Close association of a canine host is the major risk factor in humans and vulnerable individuals need to be persuaded to practice hygienic practices to minimize the risk of zoonotic infection.

In conclusion, differentiation of $S$. pseudintermedius from $S$. aureus is required to understand the epidemiology of the disease, diagnosis, clinical presentation, and further interpretation of antimicrobial resistance in the human clinical context. Suitable hygienic practices post handling of dogs and improvement of laboratory facilities would help minimize the incidence of $S$. pseudintermedius infections in humans.

Bacteriology division, Veterinary Research Institute, Peradeniya, Sri Lanka Address for correspondence: Dr Roshan Priyantha, Bacteriology division, Veterinary Research Institute,

Peradeniya, Sri Lanka. Telephone: +0094763911831 E-mail: madalagamaroshan@gmail.com

(iD https://orcid.org/0000-0002-6118-0100

Received 28 August 2021 and revised version accepted 31 December 2021. Published 7 February 2022

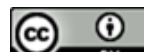

use, distribution, and reproduction in any medium, provided the original author and source are credited. 
Key words: Staphylococcus pseudintermedius, Human, Clinical infections

\section{Introduction}

The nomenclature of $S$. pseudintermedius is quite complicated, $S$. pseudintermedius, $S$. intermedius and S. delphini are collectively called S. intermedius group. ${ }^{1}$ S. pseudintermedius is a coagulase positive Gram positive coccus which colonizes $90 \%$ of healthy dogs. ${ }^{2}$ It is an opportunistic pathogen, and the most common cause of canine pyoderma, otitis externa and the second commonest cause of urinary tract infection in dogs. ${ }^{3}$ Zoonotic infections have been reported worldwide with a range of clinical presentations. ${ }^{4}$ However, reporting of this organism from human samples is rare. ${ }^{5}$ The organism is often misidentified as $S$. aureus due to cultural, morphological and biochemical similarity. ${ }^{6,7,8}$ Importantly, since $S$. aureus is the dominant pathogen in humans and both $S$. aureus and $S$. pseudintermedius are coagulase positive, laboratory misidentification is a possible reason for the low number of reports. ${ }^{6}$ Differentiation of $S$. pseudintermedius from $S$. aureus is therefore required for further identification. The objective of this overview is to highlight the epidemiology, clinical presentation, importance of laboratory tests and antimicrobial resistance of $S$. pseudintermedius in humans.

\section{Normal habitat}

The canine family is the primary reservoir of $S$. pseudintermedius. ${ }^{5}$ Although $S$. pseudintermedius is also found in cats and equines, it is primarily found in dogs, and has also been isolated in humans as either a coloniser or pathogen. Nasal colonization in methicillin resistant S. pseudintermedius (MRSP) has been reported in healthy people. ${ }^{9}$ The organism colonizes $90 \%$ of healthy dogs and their saliva contains S. pseudintermedius. ${ }^{6}$

\section{Epidemiology}

Importantly, S. pseudintermedius was isolated from healthy pet owners and other household members with no clinical disease. ${ }^{10,11} \mathrm{~S}$. pseudintermedius had been detected in veterinary surgeons who medicate dogs and cats, both as a coloniser or a pathogen. ${ }^{12,13}$ The bacterium had been isolated in veterinary health care providers, workers in vet hospitals, and also in dog owners. ${ }^{14,15}$ Therefore, isolation of the organism is not an indicator of an infection either in dogs or humans. However, detection of the organism with an associated clinical infection is evidence of an infection in dogs and humans. The history of the patient is important to find the source of this bacterial infection in human.

In addition, economic significance or outcome of $S$. pseudintermedius has not been evaluated in humans. We searched Pubmed with keywords of "Staphylococcus pseudintermedius", "dogs", and "Sri Lanka". Only one publication was found from Sri Lanka as a collaborative project with a laboratory in the Netherlands. ${ }^{2}$ Human infections caused by S. pseudintermedius have not been reported from Sri Lanka. 


\section{Pathogenesis of Staphylococcus pseudintermedius infection in humans}

The pathogenesis of human $S$. pseudintermedius infection has not been fully understood. It is assumed that similar virulence mechanisms are found in $S$. pseudintermedius and S. aureus. ${ }^{11}$ Panton valentine leukocidin (pvl), exfoliative toxins and other virulence factors such as coagulase, lipases, protein A and beta hemolysin may play a major role in the process of pathogenicity. ${ }^{16}$ S. pseudintermedius sequence type (ST) 71 which was the world epidemic clone has been observed to have high affinity and a high degree of adherence to human corneocytes. ${ }^{17}$ It is possible therefore that ST 71 may adhere to human skin more than any other ST type of $S$. pseudintermedius.

Being an opportunistic pathogen, S. pseudintermedius causes secondary infection in humans. ${ }^{17}$ The majority of clinical cases were shown among the adult population, and in patients with metabolic disease such as diabetes mellitus, or with an immunocompromised disease, and in patients with AIDS, cancers and autoimmune diseases. ${ }^{6,17-19}$ In addition, surgical interventions have been recognized as a potential risk factor in infection of a healthy human, while the clinical condition may be aggravated and worse in immunosuppressed people. ${ }^{6}$ Although human to human transmission has not been reported in S. pseudintermedius, acquiring human infection through dogs have been investigated using different molecular techniques. ${ }^{15,16}$ People who have close association with dogs have been grouped into vulnerable populations such as animal handlers, veterinarians, and veterinary technicians. ${ }^{2}$ Therefore, close friendship with dogs by immune suppressed owners or senior citizens with canine companions require extra precautions including maintaining good personal hygiene (personal observation). Similar guidelines need to be followed after surgical intervention; limited contact with companion animals is advisable.

\section{Clinical presentation of infection caused by Staphylococcus pseudintermedius}

Reporting of zoonotic infection caused by S. pseudintermedius as case studies and collection of clusters of infections has increased in the literature. ${ }^{6,7}$ A couple of papers highlighted that dog bite wounds were the commonest cause of human infection by $S$. pseudintermedius. ${ }^{19,20}$ Therefore, looking for S. pseudintermedius in dog bite wounds is required since the organism is common in the oral cavity, pharynx and saliva of dogs. ${ }^{1}$

S. pseudintermedius had been observed with skin and soft tissue infection in humans. ${ }^{6}$ A study by Somayaji $\mathrm{R}$ et al (2016) has discussed and reported the incidence of human $S$. pseudintermedius infection in Calgary, Canada, during the period 2013-15. ${ }^{6}$ The bacterium had been isolated in 18 patients who reported with skin and soft tissue infections. ${ }^{6,7}$ Most of the skin and soft tissue infections by $S$. pseudintermedius in humans were found on the lower limb. ${ }^{6}$ The annual rate in culture positivity of $S$. pseudintermedius in skin and soft tissue infections was reported as $0.025 \% .^{6}$ The patients were mostly managed as outpatients as admission to hospital was not required. ${ }^{6}$ Importantly, most cases were reported as polymicrobial infection. ${ }^{6}$ Moreover, skin infections of S. pseudintermedius were observed as common in adults or senior citizens who have close contact with a canine host. ${ }^{4,6}$

Bacteremia caused by $S$. pseudintermedius had been reported less frequently. ${ }^{6,18}$ The incidence of blood stream infection was as low as 3.48/1000 in humans. ${ }^{6}$ In the same study, the incidence of 
blood stream infection caused by $S$. aureus was $0.006 / 1000$ in humans. ${ }^{6}$ S. pseudintermedius bacteremia had been diagnosed in a 4-month-old paediatric oncology patient and a bone marrow transplanted leukaemia patient who suffered an invasive spinal infection. ${ }^{21}$ Catheter related blood stream infection has also been reported in S. pseudintermedius. ${ }^{22}$ Cleanliness and catheter care are vitally important in patients who have close contact with dogs. ${ }^{23}$ Handling of dogs needs to be avoided or extra care taken by patients who have a vascular catheter.

The first human case of $S$. pseudintermedius was reported in a patient who had a cardioverterdefibrillator implant. ${ }^{24}$ Secondary infections have been detected after an application of bone implant and surgical implant as spinal fixation devices. ${ }^{25,26}$ In addition, S. pseudintermedius had been isolated in patients who developed peritoneal dialysis-associated peritonitis. ${ }^{27}$ The organism had also been isolated in a graft-versus-host disease-related wound infection caused by a multidrug-resistant strain. ${ }^{25}$ Furthermore, S. pseudintermedius had been isolated in a 50-year-old female patient who has a history of bilateral lung transplantation. ${ }^{28}$ This patient also showed signs of sepsis, and had pneumonia, rheumatoid arthritis and severe osteoporosis. ${ }^{29} S$. pseudintermedius had been isolated from totally implantable venous access ports (TIVAP), and post-surgery purulent rhinosinusitis. ${ }^{30,31}$

Rhinosinusitis, endocarditis, prosthetic joint infection, external ear infection, urinary tract infection, catheter associated bacteremia and surgical site infections caused by $S$. pseudintermedius in humans have been reported. ${ }^{4,6,8,19,32-35}$ The organism had been isolated in a patient who had chronic sinusitis and was in close association with dogs. ${ }^{8} \quad$ S. pseudintermedius has been isolated from patients with sarcoidosis, Crohn's disease, or a history of lymphoma. ${ }^{36} S$. pseudintermedius was isolated from a patient with hepatocellular carcinoma with a urinary tract infection. ${ }^{31} \mathrm{~S}$. pseudintermedius was also isolated and identified in complicated urinary tract infections in senior citizens. ${ }^{33}$

Table 1: Biochemical tests of $S$. aureus and S. pseudintermedius

\begin{tabular}{|l|l|l|}
\hline Biochemical test & S. aureus & S. pseudintermedius \\
\hline $\begin{array}{l}\text { Colony } \\
\text { morphology }\end{array}$ & $\begin{array}{l}\text { Yellow/golden } \\
\text { colonies }\end{array}$ & $\begin{array}{l}\text { Greyish-white } \\
\text { opaque colonies }\end{array}$ \\
\hline Hemolysis & $\begin{array}{l}\text { Alpha or Beta } \\
\text { hemolysis }\end{array}$ & Beta hemolysis \\
\hline Catalase test & Positive & Positive \\
\hline $\begin{array}{l}\text { Tube coagulase } \\
\text { test }\end{array}$ & Positive & Positive \\
\hline $\begin{array}{l}\text { Slide coagulase } \\
\text { test }\end{array}$ & Positive & Varied \\
\hline $\begin{array}{l}\text { Hyaluronidase } \\
\text { test }\end{array}$ & Positive & Negative \\
\hline DNase & Positive & Positive \\
\hline VP test & Positive & Positive \\
\hline D Mannitol & Positive & Positive \\
\hline Moltose & Positive & Positive \\
\hline Trehalose & Positive & Positive \\
\hline Lactose & Positive & Positive \\
\hline $\begin{array}{l}\text { Pyrrodinyl acryl } \\
\text { amidase }\end{array}$ & Positive & Negative \\
\hline $\begin{array}{l}\text { Beta } \\
\text { galactosidase }\end{array}$ & Negative & Positive \\
\hline
\end{tabular}

\section{Laboratory diagnosis}

S. pseudintermedius is a Gram positive coccus which is readily grown on $5 \%$ sheep blood agar. ${ }^{1,17}$ It produces $1-2 \mathrm{~mm}$ greyish white colonies on $5 \%$ sheep blood agar with a double zone of hemolysis. ${ }^{35}$ The colonies of $S$. aureus are yellow on $5 \%$ sheep blood agar, with either complete or partial hemolysis. ${ }^{3}$ The differentiation of these two species on overnight blood agar plates under refrigerator conditions is challenging due to cold induced hemolysis of both species of staphylococci. Although both organisms are coagulase positive, only tube agglutination test has been recommended in $S$. pseudintermedius, while slide agglutination often shown false negative results. ${ }^{1}$ Further differentiation is 
done by a series of biochemical tests as shown in Table 1. In addition, species specific PCR (both conventional and qPCR) molecular techniques are being used to differentiate the two organisms. ${ }^{1}$

It is believed that $S$. pseudintermedius is misdiagnosed in human diagnostic laboratories due to the similar colony morphology and lack of awareness of the fairly new and canine dominant coagulase positive staphylococci. ${ }^{20}$ Consequently, underreporting of the organism and misidentification of this species is possible in diagnostic laboratories. ${ }^{6,20}$ Characteristics of this species as described above are when the organism is grown on 5\% sheep blood agar. However, diagnostic laboratories in Sri Lanka often use human blood (personal communication) and there is no available information on colony characteristics on human blood agar. A study of dog bite wounds and wounds in patients having a close association with canines with particular emphasis on $S$. pseudintermedius would be timely.

Antimicrobial resistance is a serious challenge both in human and animal medicine. ${ }^{6}$ MRSP has been isolated both in healthy and clinical patients. ${ }^{6,7}$ Importantly, MRSP and methicillin resistant $S$. aureus (MRSA) are resistant to all the beta lactam antibiotics, and resistance to other classes of antimicrobials is often found. ${ }^{3,6}$ As in MRSA, MRSP is caused by the presence of the mecA gene in $\mathrm{SCC}_{\mathrm{mec}}$ element which is usually found in a transposon. ${ }^{6,37} \mathrm{In}$ addition, multidrug resistance has been reported both in MRSP and methicillin susceptible S. pseudintermedius (MSSP) in humans. ${ }^{6,38}$ Two major epidemic clones have been reported in MRSP as sequence type (ST) 71 and ST 68, both of which have also been reported in human clinical infections. ${ }^{6}$ The antimicrobial susceptibility testing clinical break point against methicillin is different in $S$. aureus (Minimum inhibitory concentration of resistance in methicillin is $>2 \mathrm{mg} / \mathrm{l}$ ) and $S$. pseudintermedius (Minimum inhibitory concentration of resistance in methicillin is $>0.5 \mathrm{mg} / \mathrm{l}) .{ }^{39,40}$ Therefore, when the bacterium is misidentified, interpretation of methicillin resistance may be incorrect (Table 2). In addition, oxacillin with $\mathrm{NaCl}$ has been recommended in $S$. pseudintermedius instead of cefoxitin to detect methicillin resistance in the disk diffusion method. ${ }^{40,41}$ These differences in testing and interpretation of antibiotic susceptibility are a strong reason for pursuing identification of $S$. pseudintermedius in both medical and veterinary clinical microbiology laboratories.

Table: 2, Interpretation of Clinical breakpoint in $S$. aureus and $S$. pseudintermedius (Source from EUCAST clinical breakpoint 2022/01/01. CSLI ${ }^{39,40,42}$ )

\begin{tabular}{|l|l|l|l|}
\hline The organism & Description & Resistant breakpoint & Reference \\
\hline S. pseudintermedius & No clinical BP cefoxitin & $\begin{array}{l}\text { For oxacillin } \geq 0.5 \mu \mathrm{g} / \mathrm{ml} \\
(\leq 17 \mathrm{~mm} \text { for the disk } \\
\text { diffusion test })\end{array}$ & $\begin{array}{l}\text { CLSI VET 01 S3, 2015 } \\
\text { and CLSI news update } \\
2020\end{array}$ \\
\hline $\begin{array}{l}\text { S. } \text { pseudintermedius } \\
\text { S. } \text { aureus }\end{array}$ & $\begin{array}{l}\text { No BP for cefoxitin of MIC } \\
\text { determination either by cefoxitin or } \\
\text { oxacillin }\end{array}$ & $\begin{array}{l}\text { Oxacillin: } 20 \mathrm{~mm} \\
(1 \mu \mathrm{g} \text { disk })\end{array}$ & EUCAST \\
(No zone diameter for the disk diffusion & $\begin{array}{l}>2 \mu \mathrm{g} / \mathrm{ml} \text { for oxacillin and } \\
>8 \mathrm{~g} / \mathrm{ml} \text { for cefoxitin } \\
(\leq 21 \mathrm{~mm} \text { for disk diffusion } \\
\text { test })\end{array}$ & $\begin{array}{l}\text { CLSI news update } \\
2020\end{array}$ \\
\hline S. aureus & $\begin{array}{l}\text { No BP for oxacillin. BP for cefoxitin for } \\
\text { the disk diffusion test and no breakpoint } \\
\text { for MIC has been described. }\end{array}$ & Cefoxitin: $22 \mathrm{~mm}$ & EUCAST \\
\hline
\end{tabular}


Although no extensive studies have been published, S. pseudintermedius is a biofilm producer which encourages the emergence of antimicrobial resistance in an atypical environment. ${ }^{24}$

\section{Conclusion}

Differentiation of $S$. pseudintermedius from $S$. aureus is important both for laboratory interpretation and clinical decision making. S. pseudintermedius can be the cause of a risky bacterial infection when the normal immune system is not functioning and with wounds following dog bites. Multidrug resistant isolates can be a threat with severe clinical infection. Awareness of this species with improvement of laboratory diagnostic capability is required for rapid diagnosis and optimum intervention in both animals and humans.

Acknowledgement: Author would like to acknowledge the Fleming Fund programme (UK), University of Hong Kong for granting library access, Dr Wu Peng (University of HK) and all the encouragement received under the Fleming Fund Fellowship programme.

\section{References}

1 Bannoehr J, Guardabassi L. Staphylococcus pseudintermedius in the dog: taxonomy, diagnostics, ecology, epidemiology and pathogenicity. Vet. Dermatology 2012; 23:253-266, e251-252. doi:10.1111/j.1365-3164.2012. 01046.x

2 Duim B, Verstappen KMHW, Kalupahana R, et al. MRSP among dogs in the description of novel SCCmec variant. Vet. Microbiology 2018; 213: 131-148.

doi: 10.1016/j.vetmic.2017.11.022. Epub 2017 Nov 21.

3 Priyantha R, Gaunt MC, Rubin JE. Antimicrobial susceptibility of Staphylococcus pseudintermedius colonizing healthy dogs in Saskatoon, Canada. The Canadian veterinary journal 2016; 57:65-69.

4 Kmieciak W, Szewczyk EM. Are zoonotic Staphylococcus pseudintermedius strains a growing threat for humans? Folia Microbiology (Praha) 2018; 63:743-747. doi:10.1007/s12223-018-0615-2

5 Durdik P, Fedor M, Jesenek M, et al. Staphylococcus intermedius rare pathogen of acute meningitis. Int J Infect Dis 2010; 14:3, e236-238. doi: 10.1016/j.ijid.2009.08.020

6 Somayaji R, Priyantha MAR, Rubin JE, et al. Human infections due to Staphylococcus pseudintermedius, an emerging zoonosis of canine origin: report of 24 cases. Diagn Microbiol Infect Dis 2016; 85:471-476. doi: 10.1016/j.diagmicrobio.2016.05.008

7 Somayaji R, Rubin JE, Priyantha MAR, et al. Exploring Staphylococcus pseudintermedius: an emerging zoonotic pathogen? Future microbiology 2016; 11:1371-1374.

doi:10.2217/fmb-2016-0137

8 Lozano C, Rezusta A, Ferrer I, et al. Staphylococcus pseudintermedius human infection cases in Spain: Dog-to-human transmission. Vector borne and zoonotic diseases (Larchmont, N.Y.) 2017; 17: 268-270. doi:10.1089/vbz.2016.2048.

9 Rodrigues AC, Belas A, Belas A, Marques C, et al. Risk factors for nasal colonization by methicillinresistant Staphylococci in healthy humans in professional daily contact with companion animals in Portugal. Microb Drug Resist 2018; 24: 434-446. doi:10.1089/mdr.2017.0063.

10 Gómez-Sanz E, Torres C, Lozano C, et al. High diversity of Staphylococcus aureus and Staphylococcus pseudintermedius lineages and toxigenic traits in healthy pet-owning household members. Underestimating normal household contact? Comp Immunol Microbiol Infect Dis 2013; 36: 83-94. doi: 10.1016/j.cimid.2012.10.001 
11 Gómez-Sanz E, Torres C, Ceballos S, et al. Clonal dynamics of nasal Staphylococcus aureus and Staphylococcus pseudintermedius in dog-owning household members. Detection of MSSA ST(398). PLoS One 2013; 8:e69337. doi: 10.1371/journal.pone.0069337

12 Tabatabaei S, Nijafifar A, Akari B, et al. Genetic characterisation of methicillin-resistant Staphylococcus aureus and Staphylococcus pseudintermedius in pets and veterinary personnel in Iran: new insights into emerging methicillin-resistant $S$. pseudintermedius (MRSP). Journal of global antimicrobial resistance 2019;16: 6-10. doi: 10.1016/j.jgar.2018.08.022

13 Paul NC, Moodley A, Ghibaudo G, et al. Carriage of methicillin-resistant Staphylococcus pseudintermedius in small animal veterinarians: indirect evidence of zoonotic transmission. Zoonoses Public Health 2011; 58: 533-539. doi:10.1111/j.1863-2378.2011.01398.x

14 Worthing KA, Brown J, Gerber L, et al. Methicillin-resistant staphylococci amongst veterinary personnel, personnel-owned pets, patients and the hospital environment of two small animal veterinary hospitals. Vet Microbiol 2018; 223: 79-85. doi: 10.1016/j.vetmic.2018.07.021

15 Wettstein RK, Rothenager E, Brodard I, et al. Nasal carriage of methicillin-resistant Staphylococcus aureus (MRSA) among Swiss veterinary health care providers: detection of livestock and healthcare associated clones. Schweiz Arch Tierheilkd 2014; 156: 317-325. doi:10.1024/0036-7281/a000601

16 Soedarmanto I, Kambar T, Ulbegi $\mathrm{MH}$, et al. Genetic relatedness of methicillin-resistant Staphylococcus pseudintermedius (MRSP) isolated from a dog and the dog owner. Res Vet Sci 2011; 91: e25-27. doi: 10.1016/j.rvsc.2011.01.027

17 Latronico F, Moodely A, Neilsen SS, et al, Enhance adherence of methicillin resistant Staphylococcus pseudintermedius sequence type 71 to canine and human corneocyte. Veterinary Research 2014; 45: 701-707 doi.org/10.1186/1297-9716-45-70

18 Winn W. Koneman's Color Atlas and Textbook of Diagnostic Microbiology 2006; Ch. 12, 623-671 (Lippincott Williams \& Wilkins, 2006).

19 Bhooshan S, Negi V, Khatri PK. Staphylococcus pseudintermedius: an undocumented, emerging pathogen in humans. GMS Hyg Infect Control 2020; 15:Doc32. doi:10.3205/dgkh000367

20 Borjesson S, Gomez-Sanz E, Ekstrom K, et al, Staphylococcus pseudintermedius can be misdiagnosed as Staphylococcus aureus in humans with dog bite wounds. Eur J Clin Microbiol Infect Dis 2015; 34: 839-844. doi:10.1007/s10096-014-2300-y

21 Blondeau LD, Rubin JE, Deneer H, et al. Bacteremia with Staphylococcus pseudintermedius in a 4 month old pediatric oncology patient. J Chemother2020; 32: 260-262. doi:10.1080/1120009x.2020.1773627

22 Diaz MA, Gardner LB, Libertin CR. Staphylococcus pseudintermedius catheter-related bloodstream infection after exposure to domestic dogs and a cat. BMJ Case Rep 12. doi:10.1136/bcr-2019-231489 (2019).

23 Maali Y, Badiu C, Martin C, et al. Understanding the virulence of Staphylococcus pseudintermedius: A major role of pore-forming toxins. Frontiers in Cellular and Infection Microbiology 2018; 8. doi:10.3389/fcimb.2018.00221

24 Hoovels LV, Vankeerberghen A, Boel A, et al. First case of Staphylococcus pseudintermedius infection in a human. Journal of Clinical Microbiology 2006; 44: 4609-4612. doi:10.1128/JCM.01308-06

25 Savini V, Barabarini D, Polokoswsca K, et al. Methicillin-resistant Staphylococcus pseudintermedius infection in a bone marrow transplant recipient. $J$ Clin Microbiol 2013; 51:1636-1638. doi:10.1128/jcm.03310-12

26 Darlow CA, Paidakakos N, Sikander M. A spinal infection with Staphylococcus pseudintermedius. BMJ Case Rep 2017; 2017:bcr-2017-221260. doi:10.1136/bcr-2017-221260

27 Dahbour L, Gibs J, Coletta C, et al. Peritoneal dialysis zoonotic bacterial peritonitis with Staphylococcus pseudintermedius. Case Reports in Nephrology and Dialysis 2020; 10:65-70, doi: $10.1159 / 000508126$ 
28 Small C, Beatty N, Helou, EIG. Staphylococcus pseudintermedius bacteremia in a lung transplant recipient exposed to domestic pets. Cureus 2021; 13:e14895. doi:10.7759/cureus. 14895

29 Blondeau LD, Sanch S, Sauda DJ, et al. Recovery of borderline oxacillin-resistant Staphylococcus pseudintermedius (BORSP) from bone and soft tissue of a rheumatoid arthritis patient with severe osteoporosis: transmission from the family dog. $J$ Chemother 2021: 1-6, doi:10.1080/1120009x.2021.1879581

30 Nomoto, H. Katsuna S, Makamura K, et al. Totally implantable venous access port infection caused by Staphylococcus pseudintermedius: Possible transmission from a companion dog to a human. J Infect Chemother 26, 1305-1308, doi:10.1016/j.jiac.2020.07.011

31 Kitagawa $\mathrm{H}$, Hisatsune $\mathrm{J}$, Ohje $\mathrm{H}$, et al. Implanted port catheter system infection caused by Methicillin-resistant Staphylococcus pseudintermedius ST71-SCCmec type III. Internal medicine (Tokyo, Japan); 2021: 60:2337-2340. doi:10.2169/internalmedicine.5579-20

32 Riegel P, Jesel ML, Leventie B, et al. Coagulase-positive Staphylococcus pseudintermedius from animals causing human endocarditis. Int J Med Microbiol 2021; 301: 237-239., doi: 10.1016/j.ijmm.2010.09.001.

33 Subedi P, Syed MP, Kate Y, Staphylococcus pseudintermedius: a common zoonotic pathogen causing postprocedural urosepsis in humans. BMJ Case 2021; Rep $14 . \quad$ doi:10.1136/bcr-2020-238170

34 Ference, EH. Danielian A, Kim HW, et al. Zoonotic Staphylococcus pseudintermedius sinonasal infections: risk factors and resistance patterns. Int Forum Allergy Rhinol 9, 724-729, doi:10.1002/alr.22329

35 Gagetti P, Errecalde L, Wattam AR, et al. Characterization of the first mecA-positive multidrugresistant Staphylococcus pseudintermedius isolated from an Argentinian patient. Microb Drug Resist 2020: 26, 717-721. doi:10.1089/mdr.2019.0308

36 Kuan EC, Yoon AJ, Vijayan T, et al. Canine Staphylococcus pseduintermedius sinosal infection in human host. Int Forum Allergy Rhinol 2016; 6(7):710-715, doi: 10.1002/alr.21732.

37 Humphries RM, Wu MT, Westblade LF, et al. In vitro antimicrobial susceptibility of Staphylococcus pseudintermedius isolates of human and animal origin. J Clin Microbiol 2016; 54:1391-1394. doi:10.1128/jcm.00270-16

38 Stegmann R, Burnens A, Maranta CA, et al. Human infection associated with methicillin-resistant Staphylococcus pseudintermedius ST71. J Antimicrob Chemother 2010; 65: 2047-2048, doi:10.1093/jac/dkq241

39 CLSI Subcommittee on Antimicrobial Susceptibility Testing CLSI AST News Update. (2020) clsi.org/media/3486/clsi_astnewsupdate_january2020.pdf.

40 CLSI. Performance Standards for Antimicrobial Disk and Dilution Susceptibility Tests for Bacteria isolated From Animals: Approved Standard-Fourth Edition. (2013).

41 Wu MT, Burnham CMD, Westblade LF, et al. Evaluation of oxacillin and cefoxitin disk and MIC breakpoints for prediction of methicillin resistance in human and veterinary isolates of Staphylococcus intermedius Group. J Clin Microbiol 2016; 54: 535-542. doi:10.1128/jcm.02864-15

42 Skov R, Vagar A, Matuschek V, et al. EUCAST disc diffusion criteria for the detection of mecAMediated $\beta$-lactam resistance in Staphylococcus pseudintermedius: oxacillin versus cefoxitin. Clinical Microbiology and Infection: 2020; 26: 122.e121-122.e126.

doi: 10.1016/j.cmi.2019.05.002 